\title{
On One Nonlinear Boundary-Value Problem in Kinetic Theory of Gases
}

\author{
A.Kh. Khachatryan \\ Armenian National Agrarian University \\ 74 Teryan St., Yerevan, 0009, Armenia \\ E-mail: Aghavard@hotbox.ru
}

Kh.A. Khachatryan and T.H. Sardaryan

\author{
Institute of Mathematics of National Academy of Sciencesof Armenia \\ 24/5 Baghramyan Ave., Yerevan 0019, Armenia \\ E-mail: Khach82@rambler.ru \\ Sardaryan.tigran@gmail.com
}

Received September 9, 2013, revised February 5, 2014

In the paper, the solvability of one nonlinear boundary-value problem arising in kinetic theory of gases is studied. We prove the existence of global solvability of a boundary-value problem in the Sobolev space $W_{\infty}^{1}\left(\mathbb{R}^{+}\right)$. The limit of the solution is found by using some a'priori estimations. For the case of power nonlinearity, the uniqueness of the solution in a certain class of functions is proved. Some examples illustrating the obtained results are given.

Key words: boundary-value problem, monotony, nonlinear integral equation, iteration, limit of solution.

Mathematics Subject Classification 2010: 45G05, 35G55.

\section{Introduction. Statement of the Problem}

The paper is devoted to the following nonlinear boundary-value problem:

$$
\begin{gathered}
\pm s \frac{\partial \varphi^{ \pm}(x, s)}{\partial x}+\varphi^{ \pm}(x, s)=G(U(x)), \quad x>0, s>0 \\
\varphi^{+}(0, s)=G_{1}\left(\int_{0}^{\infty} Q(s, p) \varphi^{-}(0, p) d p\right)
\end{gathered}
$$

This work was supported by State Committee of Science MES RA in the frame of the research project CS 13-1A068.

(c) A.Kh. Khachatryan, Kh.A. Khachatryan, and T.H. Sardaryan, 2014 


$$
\varphi^{-}(x, s)=o\left(e^{\frac{x}{s}}\right), \quad x \rightarrow+\infty
$$

where

$$
U(x)=\frac{1}{\sqrt{\pi}} \int_{0}^{\infty} e^{-p^{2}}\left[\varphi^{+}(x, p)+\varphi^{-}(x, p)\right] d p .
$$

The functions $G$ and $G_{1}$ describe the nonlinear dependence in the right-hand side of integro-differential equation (1) and the nonlinear dependence of boundary condition (2), respectively. The function $Q(s, p)$ describes the general law of reflection and possesses the substochasticity property

$$
Q(s, p) \geq 0,(s, p) \in \mathbb{R}^{+} \times \mathbb{R}^{+}, \int_{0}^{\infty} Q(s, p) d p \leq 1 .
$$

Boundary-value problem (1)-(4) can be derived from the Boltzmann equation within the framework of one model suggested in [5] and it has important applications in kinetic theory of gases (see [1-6] and references therein). By means of equations (1), (4) with boundary value conditions (2), (3), the flow of a gas with average mass velocity $U(x)$ in a half space $x>0$ bounded by the plate wall $x=0$ is described.

Problem (1)-(4) in a standard way can be reduced to the nonlinear integral equation

$$
U(x)=\mu(x, U)+\int_{0}^{\infty} K(x-t) G(U(t)) d t
$$

where

$$
\begin{gathered}
\mu(x, U)=\frac{1}{\sqrt{\pi}} \int_{0}^{\infty} e^{-\frac{x}{s}} e^{-s^{2}} G_{1}\left[\int_{0}^{\infty} Q(s, p) d p \int_{0}^{\infty} e^{-\frac{t}{p}} G(U(t)) \frac{d t}{p}\right] d s, \\
K(x)=\frac{1}{\sqrt{\pi}} \int_{0}^{\infty} e^{-\frac{|x|}{s}} e^{-s^{2}} \frac{d s}{s} .
\end{gathered}
$$

In the linear case, where $G(x) \equiv x, \quad G_{1}(x) \equiv x$, the investigation of the problem (1)-(4) was carried out in a number of works (see [1,6] and references therein).

In all the papers mentioned, the average mass velocity possesses asymptotics $O(x)$ when $x$ tends to $+\infty$.

In the case of the linear law of reflection (i.e., where $G_{1}(x) \equiv x$ ), in [5], by imposing some natural conditions on the function $G$, it was shown that there exists qualitative difference between the solutions for the linear $(G(x) \equiv x)$ and 
nonlinear cases. In the linear case, the solution has a linear growth away from the wall, while in the nonlinear case it has a bounded solution with the finite limit at infinity.

In the present paper, the question of solvability of nonlinear integral equation (6) is considered. Under certain conditions imposed on the functions $G, G_{1}$ (see below), the existence of a positive bounded solution of equation (6) is proved. The limit of the solution at infinity representing isothermal sliding coefficient is found. In the case of power nonlinearity (i.e., where $G(x)=x^{\alpha}$ ), the uniqueness of the solution in a certain class of functions is proved. Some examples of nonlinearity illustrating the obtained results are given.

\section{Basic Results}

Let $G_{0}(z)$ be a real measurable function defined on the set $(-\infty,+\infty)$ and satisfying the following conditions:

a) The numbers $\eta$ and $\xi$ are assumed to be the first positive roots of the equations $G_{0}(z)=z$ and $G_{0}(z)=2 z$, respectively, and besides $2 \xi<\eta$,

b) $G_{0} \in C[0, \eta], G_{0} \uparrow$ on the interval $[\xi, \eta]$.

Here are the examples of the above function:

$$
\begin{aligned}
& \text { 1) } G_{0}(z)=z^{p} ; 0<p<1, \xi=\left(\frac{1}{2}\right)^{\frac{1}{1-p}} ; \eta=1, \\
& \text { 2) } G_{0}(z)=e^{z-1}, \xi \approx 0,2, \eta=1 .
\end{aligned}
$$

Below, assuming that the initial function $G(z)$ is the local majorant for the function $G_{0}(z), \eta$ is a fixed point for the function $G_{1}(z)$, and imposing some natural conditions on the functions $G$ and $G_{1}$, we will prove global solvability of equation (6) in the space of essentially bounded functions.

Moreover, by using special a'priori estimations, the limit of the solution at infinity will be found. In one important particular case, where $G(z)=z^{p}, 0<$ $p<\frac{1}{2}$ and the function $G_{1}$ additionally satisfies the Lipschitz condition

$$
\left|G_{1}\left(z_{1}\right)-G_{1}\left(z_{2}\right)\right| \leq \alpha\left|z_{1}-z_{2}\right|, \quad \alpha \in(0 ; 1], \quad z_{1} ; z_{2} \in\left[\left(\frac{1}{2}\right)^{\frac{1}{1-p}}, 1\right],
$$

the uniqueness of the solutions in a certain class of functions will be proved.

The following results are true:

Theorem 1. Let the functions $G(s)$ and $G_{1}(z)$ satisfy the following conditions:

$\left.i_{1}\right) \quad G(z) \geq G_{0}(z), \quad z \in[\xi, \eta], G(\eta)=G_{1}(\eta)=\eta$, 
$\left.i_{2}\right) \quad G, G_{1} \uparrow$ on the interval $[\xi, \eta]$ and $G_{1}(z) \geq 0, z \in[\xi, \eta], G ; G_{1} \in C[0 ; \eta]$

and the function $Q(s, p)$ satisfy condition (5).

Then equation (6) has a positive essentially bounded solution $U(x)$, and besides

$$
\lim _{x \rightarrow \infty} U(x)=\eta
$$

Theorem 2. Let $G(z)=z^{p} ; p \in\left(0, \frac{1}{2}\right)$, and the function $G_{1}$ satisfy the conditions of Theorem 1 and condition (11). Then equation (6) has a unique solution in the following class of measurable functions:

$$
P=\left\{f(x):\left(\frac{1}{2}\right)^{\frac{1}{1-p}} \leq f(x) \leq 1, x \in(0,+\infty)\right\}
$$

\section{Proof of the Main Results}

P r o o f of Theorem 1. With the help of equation (6), we consider the auxiliary Hammerstein type nonlinear integral equation

$$
\varphi(x)=\int_{0}^{\infty} K(x-t) G_{0}(\varphi(t)) d t, \quad x>0,
$$

with respect to an unknown measurable real function $\varphi(x)$, where kernel $K$ is given by formula (8).

From (8), it follows that

$$
K(-x)=K(x), \quad x \geq 0 \text { and } \int_{-\infty}^{+\infty} K(x) d x=1 .
$$

In [4], not only the existence of positive solution $\varphi(x)$ for equations (15), (16) was proved, but also the following properties were established:

$$
\lim _{x \rightarrow \infty} \varphi(x)=\eta ; \quad \varphi(x) \geq \xi, \quad x \geq 0 .
$$

Let us consider the iteration for basic equation (6) taking into account (7),

$$
\begin{gathered}
U_{n+1}(x)=\frac{1}{\sqrt{\pi}} \int_{0}^{\infty} e^{-s^{2}} e^{-\frac{x}{s}} G_{1}\left(\int_{0}^{\infty} Q(s, p) \frac{d p}{p} \int_{0}^{\infty} e^{-\frac{t}{p}} G\left(U_{n}(t)\right) d t\right) d s \\
+\int_{0}^{\infty} K(x-t) G\left(U_{n}(t)\right) d t
\end{gathered}
$$




$$
U_{0}(x)=\varphi(x), \quad n=0 ; 1 ; 2 ; \ldots, x \geq 0 .
$$

Due to monotony of the functions $G$ and $G_{1}$ on the interval $[\xi, \eta]$, it is easy to check that

a) $U_{n}(x) \uparrow$ in $n$,

b) the functions $U_{n}(x)$ are measurable on the set $\mathbb{R}^{+} ; n=0 ; 1 ; 2, \ldots$.

Below we prove that

$$
U_{n}(x) \leq \eta, \quad n=0 ; 1 ; 2, \ldots
$$

In fact, in the case $n=0$, inequality (20) is obvious because of $U_{0}(x)=\varphi(x), \varphi(x) \uparrow$ on $\mathbb{R}^{+}$and $\lim _{x \rightarrow \infty} \varphi(x)=\eta$.

We assume that inequality (20) takes place for some $n \in \mathbb{N}$. Then in view of (12) and (5), from (18) we get

$$
\begin{aligned}
U_{n+1}(x) \leq & \frac{1}{\sqrt{\pi}} \int_{0}^{\infty} e^{-s^{2}} e^{-\frac{x}{s}} G_{1}\left(\eta \int_{0}^{\infty} Q(s, p) d p\right) d s+\eta \int_{0}^{\infty} K(x-t) d t \\
& \leq \frac{1}{\sqrt{\pi}} \int_{0}^{\infty} e^{-s^{2}} e^{-\frac{x}{s}} G_{1}(\eta) d s+\eta \int_{-\infty}^{x} K(y) d y \\
& =\eta\left(\frac{1}{\sqrt{\pi}} \int_{0}^{\infty} e^{-s^{2}} e^{-\frac{x}{s}} d s+\int_{-\infty}^{x} K(y) d y\right) \equiv J
\end{aligned}
$$

It is easy to verify that

$$
\int_{x}^{\infty} K(y) d y=\frac{1}{\sqrt{\pi}} \int_{0}^{\infty} e^{-s^{2}} e^{-\frac{x}{s}} d s,
$$

therefore $J=\eta$, and hence $U_{n+1} \leq \eta$.

Thus, the sequence of measurable functions $\left\{U_{n}(x)\right\}_{n=0}^{\infty}$ has a pointwise limit as $n \rightarrow+\infty$. By B. Levi's theorem, the function $U(x)=\lim _{n \rightarrow \infty} U_{n}(x)$ satisfies equation (6). From (18)-(20), it also follows that

$$
\varphi(x) \leq U(x) \leq \eta, \quad x \in \mathbb{R}^{+} .
$$

As $\lim _{x \rightarrow \infty} \varphi(x)=\eta$, then in view of $(21)$, we immediately get

$$
\lim _{x \rightarrow \infty} U(x)=\eta
$$

The theorem is proved. 
P r o o f of Theorem 2. We assume the opposite. Let equation (6) have two solutions from $P$. We denote their difference by $\Delta U=U^{1}-U^{2} ; U^{j} \in P, j=1,2$. Then from (6), taking into account (11) and $G(z)=z^{p}, p \in\left(0, \frac{1}{2}\right)$, we have

$$
\begin{gathered}
\Delta U(x)=\frac{1}{\sqrt{\pi}} \int_{0}^{\infty} e^{-s^{2}} e^{-\frac{x}{s}} \\
\times\left[G_{1}\left(\int_{0}^{\infty} Q(s, p) \int_{0}^{\infty} e^{-\frac{t}{p}} G\left(U^{1}(t)\right) \frac{d t d p}{p}\right)-G_{1}\left(\int_{0}^{\infty} Q(s, p) \int_{0}^{\infty} e^{-\frac{t}{p}} G\left(U^{2}(t)\right) \frac{d t d p}{p}\right)\right] d s \\
+\int_{0}^{\infty} K(x-t)\left[G\left(U^{1}(t)\right)-G\left(U^{2}(t)\right)\right] d t
\end{gathered}
$$

By the Lagrange theorem, it is easy to verity that

$$
\text { if }\left(\frac{1}{2}\right)^{\frac{1}{1-p}} \leq x_{1}, \quad x_{2} \in 1, \quad \text { then }\left|x_{1}^{p}-x_{2}^{p}\right| \leq 2 p\left|x_{1}-x_{2}\right| .
$$

Using (24) and (5), from (23) we obtain

$$
\begin{aligned}
& |\Delta U(x)| \leq \frac{\alpha}{\sqrt{\pi}} \int_{0}^{\infty} e^{-s^{2}} e^{-\frac{x}{s}} \int_{0}^{\infty} Q(s, p) \int_{0}^{\infty} e^{-\frac{t}{p}}\left|G\left(U^{1}(t)\right)-G\left(U^{2}(t)\right)\right| \frac{1}{p} d t d p d s \\
& \quad+\int_{0}^{\infty} K(x-t)\left|G\left(U^{1}(t)\right)-G\left(U^{2}(t)\right)\right| d t \\
& \leq\left[\frac{\alpha}{\sqrt{\pi}} 2 p \int_{0}^{\infty} e^{-s^{2}} e^{-\frac{x}{s}} \int_{0}^{\infty} Q(s, p) d p d s+2 p \int_{-\infty}^{x} K(y) d y\right] \sup _{t \geq 0}\left|U^{1}(t)-U^{2}(t)\right| \\
& \leq\left[2 p \alpha \int_{x}^{\infty} K(y) d y+2 p \int_{-\infty}^{x} K(y) d y\right] \sup _{t \geq 0}|\Delta U(t)| \leq 2 p \sup _{t \geq 0}|\Delta U(t)| .
\end{aligned}
$$

Hence,

$$
(1-2 p) \sup _{t \geq 0}|\Delta U(t)| \leq 0 .
$$

As $p \in\left(0, \frac{1}{2}\right)$, then due to $(25)$ we obtain that $\Delta U(t)=0$ almost everywhere on $(0 ;+\infty)$, therefore $U^{1}(t)=U^{2}(t)$ almost everywhere on $(0 ;+\infty)$. The theorem is proved. 
E x a $\mathrm{m} \mathrm{p} \mathrm{l}$ e s. As the function $G(z)$ can be chosen, for the examples of the functions $G_{0}$ see (9) and (10). However, we also give an example different from $G_{0}$,

$$
G(z)=G_{0}(z)+\frac{\eta}{\pi} \frac{\sin ^{2} G_{0}(z) \pi}{\eta} .
$$

Here are the examples of the functions $G_{1}(z)$ :

$$
\begin{aligned}
& \text { a) } G_{1}(z)=e^{z-1} ; \eta=1 ; \alpha=1, \\
& \text { b) } G_{1}(z)=\eta-\beta \tilde{G}(\eta-z) ; \beta \in\left(0, \min \left(1 ; \frac{1}{\max _{\xi \leq z \leq \eta} \tilde{G}^{\prime}(z)}\right)\right] \text {, }
\end{aligned}
$$

where $\tilde{G}(\eta)=\eta, \tilde{G} \uparrow$ on $[\xi, \eta], \max _{\xi \leq z<\eta} \tilde{G}^{\prime}(z)<+\infty, \tilde{G}(z) \geq 0, z \in[\xi, \eta]$.

For example, if $\tilde{G}(z)=z^{2}$, then $\eta=1$ and $G_{1}(z)=1-\beta(1-z)^{2}, \beta \in\left(0, \frac{1}{2}\right]$.

$\mathrm{R}$ e $\mathrm{m}$ a $\mathrm{r} \mathrm{k}$. It should be noted that the solution of initial boundary value problem (1)-(4) belongs to the space $W_{\infty}^{1}\left(\mathbb{R}^{+}\right)$in $x$.

Thus, from (1)-(3), we get

$$
\begin{gathered}
\varphi^{+}(x, s)=C(s) e^{-\frac{x}{s}}+\int_{0}^{x} e^{-\frac{(x-t)}{s}} G(U(t)) \frac{d t}{s}, \\
\varphi^{-}(x, s)=\int_{x}^{\infty} e^{-\frac{(t-x)}{s}} G(U(t)) \frac{d t}{s},
\end{gathered}
$$

where

$$
C(s)=G_{1}\left(\int_{0}^{\infty} Q(s, p) \frac{d p}{p} \int_{0}^{\infty} e^{-\frac{t}{p}} G(U(t)) d t\right) .
$$

As $U \in L_{\infty}(0,+\infty)$, then from (26), (27) it follows that for each fixed $s \in(0,+\infty)$,

$$
\varphi^{ \pm}(x, s) \in W_{\infty}^{1}\left(\mathbb{R}^{+}\right) .
$$

\section{References}

[1] C. Cercignani, The Boltzmann Equation and its Applications. Springer-Verlag, New York, 1988.

[2] M.M.R. Williams, Mathematical Methods in Particle Transport Theory. Butterworth, London, 1971. 
[3] C. Villani, Cereignami's Conjecture is Sometimes True and Always Almost True Communications in Mathematical Physics. - Israel J. Math. 234 (2003), No. 3, 455-490.

[4] A.Kh. Khachatryan and Kh.A. Khachatryan, On an Integral Equation with Monotonic Nonlinearity. - Memoirs on Differential Equations and Mathematical Physics 51 (2010), 59-72.

[5] A.Kh. Khachatryan and Kh.A. Khachatryan, Qualitative Difference between Solutions for a Model of the Boltzmann Equation in the Linear and Nonlinear Cases. J. Theor. Math. Phys. 172 (2012), No 3, 1315-1320.

[6] N.B. Engibarian and A.Kh. Khachatryan, Exact Linearization of the Sliding Problem for a Dilute Gas in the Bhatnagar-Gross-Krook model. — J. Theor. Math. Phys. 125 (2000), No 2, 239-342. 